\title{
Percepção dos Profissionais de Saúde Acerca da Síndrome Metabólica
}

\author{
Giovanna Costa Falcãoํㅜ, Tatiana Evangelista da Silva Rocha²
}

\begin{abstract}
RESUMO
O objetivo deste estudo foi compreender a percepção dos profissionais de saúde acerca da Síndrome Metabólica. Trata-se de uma pesquisa qualitativa, descritiva, exploratória e de campo, realizada com profissionais de saúde lotados em dois Centros de Saúde da Comunidade do município de Palmas/Tocantins. A coleta de dados foi no período de abril a junho de 2018. As entrevistas foram conduzidas por um único pesquisador, que utilizou um questionário semiestruturado, posteriormente analisado pelo método de Análise de Conteúdo. Conforme semelhança das falas, foram agrupadas para análise das entrevistas as categorias profissionais de enfermagem e medicina, agente comunitário de saúde e técnico de enfermagem, posto que o primeiro grupo destacou mais a fundo o tema e demonstrou menos estranheza acerca do termo, porém ainda sem aprofundamento conforme a literatura. Para o mesmo fim, os resultados foram divididos em categorias, sendo elas: conceito e diagnóstico, tratamento, fatores de risco e ações de prevenção. Os profissionais de nível superior demonstraram maior compreensão acerca da Síndrome Metabólica, porém ainda nota-se a necessidade de maior esclarecimento sobre o assunto para que a importância desta condição seja reconhecida. Desta forma, haverá uma prestação de assistência adequada aos pacientes, evitando o surgimento de novos casos de síndrome metabólica e que doenças crônicas se instalem, causando consequências econômicas e sociais para o país.
\end{abstract}

Palavras-chave: Síndrome Metabólica. Fatores de risco. Promoção da saúde. Atenção Primária à Saúde.

\section{PERCEPTION OF HEALTH PROFESSIONALS ABOUT METABOLIC SYNDROME ABSTRACT}

\section{ABSTRACT}

The objective of this study was to understand the perception of the health professionals around metabolic syndrome. It is a qualitative research, descriptive, exploratory and of area, performed with health professionals manned in two Community Health Centers in Palmas/ Tocantins, through interview by using a semi-structured questionnaire being analyzed by the Content Analysis methods. According to the speech similarity it was grouped to the interview analysis the professional categories of nursing and medicine, community health agent and nurse technician, since the first group has stood out further about the topic and shown less strangeness around the term, however still without further development according to literature. To the same purposes, the results were divided in categories: concept and diagnosis, treatment, risk factors, preventive actions. The higher education professionals demonstrated greater understanding around metabolic syndrome, but still notes that it is necessary greater understanding about the topic, to the real importance of this condition be recognized. In this way, there will be suitable provision of assistance to the patients, avoiding the emergence of new cases of metabolic syndrome and chronic diseases get settled, causing economic and social consequences to the country.

Keywords: Metabolic Syndrome. Risk factors. Health promotion. Primary Health Care.

RECEBIDO EM: 5/4/2019

MODIFICAÇÕES SOLICITADAS EM: 8/11/2019

ACEITO EM: 5/3/2020

Nutricionista. Residente em Saúde da Família e Comunidade pela Fundação Escola de Saúde Pública de Palmas. http://lattes.cnpq.br/3930804984058698. https://orcid.org/0000-0002-1495-8717. giovannacfalcao@gmail.com

${ }^{2}$ Nutricionista Doutora em Ensino da Saúde. Professora do curso de Nutrição da Universidade Federal do Tocantins. http://lattes.cnpq.br/9855752395939964. https://orcid.org/0000-0002-9740-7987. tatiana.evangelista@mail.uft.edu.br 


\section{INTRODUÇÃO}

A Síndrome Metabólica (SM) é definida por um grupo de fatores de risco cardiovascular que reflete a transição epidemiológica em que passa o Brasil e o mundo atualmente (SBC, 2005). Existem várias definições, sendo as mais utilizadas para diagnóstico aquelas preconizadas pela Organização Mundial de Saúde (OMS), International Diabetes Federation (IDF) e National Cholesterol Education Program's Adult Treatment Panel III (NCEP-ATP III), a última recomendada pela I Diretriz Brasileira de Diagnóstico e Tratamento da Síndrome Metabólica (I-DBSM) (WHO, 1999; SBC, 2005; ABESO, 2016).

Apesar de vários critérios existentes, nota-se uma concordância entre eles, tendo como maiores fatores para o desenvolvimento da SM a obesidade abdominal, a glicemia e a pressão arterial aumentadas e a dislipidemia. Estes aumentam a chance de desenvolvimento de condições crônicas como doenças cardiovasculares (DCV) e Diabetes Mellitus (DM), posto que a associação da síndrome com as DCVs aumenta a mortalidade geral em 1,5 vezes, e cardiovascular 2,5 vezes (SBC, 2005; IDF, 2006).

Estima-se que cerca de $20 \%-25 \%$ da população do mundo tenha SM, e a prevalência geral demonstrada em alguns estudos nos países da América Latina foi de 24,9\% (IDF, 2006; JARAMILLO et al., 2014). Esta prevalência encontra-se em avanço em diversas populações, podendo ser relacionada ao aumento da obesidade, com associação a hábitos alimentares inadequados e alto consumo de alimentos ricos em açúcares e gorduras, e ao sedentarismo (SBC, 2005; CASTANHO et al., 2013; SAAD et al., 2014).

De acordo com a Vigilância de Fatores de Risco e Proteção para Doenças Crônicas por Inquérito Telefônico (Vigitel) de 2016, 53,8\% e 18,9\% da população adulta brasileira encontra-se com excesso de peso e obesidade, respectivamente (BRASIL, 2017a). De acordo com o Sistema de Vigilância Alimentar e Nutricional (SISVANweb), por meio de relatório gerado, no município de Palmas $34,8 \%$ da população adulta avaliada em 2016 encontrava-se com sobrepeso e 22,9\% com obesidade (BRASIL, 2016a). Da mesma forma comportam-se os resultados obtidos em outras pesquisas, demonstrando aumento no excesso de peso e obesidade nos últimos anos desde a infância até a fase adulta (BRASIL, 2014a, 2016b).

Ainda segundo o Vigitel (BRASIL, 2017a), as pessoas diagnosticadas com hipertensão arterial sistêmica (HAS) foram 27,7\% e 8,9\% DM. Considerando a DM em âmbito mundial, sabe-se que aproximadamente
382 milhões de pessoas possuem a doença, podendo chegar a 471 milhões em 2035 em razão do envelhecimento da população, aumento da obesidade e sedentarismo (SBD, 2017). Esses indicadores mostraram-se aumentados anualmente quando comparados às pesquisas realizadas nos anos anteriores, demonstrando a importância da realização de ações de prevenção e promoção da saúde, evitando, assim, que tais doenças se instalem (BRASIL, 2017a).

Considerando a nova Política Nacional de Atenção Básica (PNAB), a atenção primária à saúde (APS) consiste no contato preferencial dos indivíduos, família e comunidade aos serviços de saúde, buscando atendê-los de forma integral, considerando sua singularidade, por meio da promoção, prevenção, diagnóstico, tratamento, reabilitação, redução de danos e manutenção da saúde (BRASIL, 2017b).

Pensando nisso e no aumento das doenças crônicas não transmissíveis (DCNTs) no Brasil, a atenção básica do município de Palmas está em processo de introdução de um novo modelo de atenção - o Modelo de Atenção às Condições Crônicas (MACC) -, sendo este já instituído em outros locais, como em Curitiba/PR (MOYSÉS; FILHO; MOYSÉS, 2012). Este Modelo no município terá como foco inicial a SM.

O MACC corrobora o presente estudo por chamar a atenção dos profissionais acerca dessas condições e sua importância, citando, ainda, em alguns dos seus níveis, a relevância de ações de promoção e prevenção dos fatores de risco, além de propor mudanças na atenção a essas doenças (MENDES, 2012).

Diante do exposto, faz-se necessária a constante atualização dos profissionais de saúde acerca do tema, sabendo diagnosticar, compreender e identificar os fatores de risco associados às efetivas ações de prevenção estabelecidas. Deste modo, compreender a percepção dos profissionais se faz relevante, pois, assim, verifica-se como está o processo de aprendizagem e se é preciso uma atualização acerca do tema, tendo como consequência uma melhor assistência ao paciente.

\section{MATERIAIS E MÉTODOS}

O presente estudo trata-se de uma pesquisa qualitativa, descritiva, exploratória e de campo. A abordagem qualitativa foi utilizada por se aplicar ao estudo da história, das relações, das representações, das crenças, das percepções e das opiniões que os entrevistados fazem a respeito do seu saber (BARDIN, 2011; MINAYO, 2014). 
A Portaria no 518, de 14 de junho de 2016, em seu artigo 6으, define que as Unidades de Saúde da Família passam a ser denominadas Centro de Saúde da Comunidade (CSC), portanto serão assim referidas no presente estudo. A pesquisa foi realizada em dois CSC do município de Palmas do Estado do Tocantins.

O município foi dividido em oito territórios de saúde, de acordo com a portaria supracitada. Os CSCs participantes da pesquisa estão localizados no território Krahô, estando geograficamente na região central de Palmas. Este foi selecionado de forma aleatória, posto que não se tem dados públicos das demandas estratificadas por território no município, porém sabe-se que os casos de sobrepeso, obesidade e demais DCNTs têm aumentado de forma geral nos últimos anos (BRASIL, 2016a).

Os CSCs selecionados possuem, em cada um, três equipes de Saúde da Família (ESF), três equipes de Saúde Bucal (ESB) e um Núcleo Ampliado de Saúde da Família (NASF-AB). A amostra foi composta por 16 profissionais de saúde distribuídos entre as ESFs, lotados nesses CSCs, sendo eles três enfermeiros, cinco médicos, quatro técnicos de enfermagem e quatro agentes comunitários de saúde.

Após aprovação pelo Comitê de Ética em Pesquisa do Centro Universitário Luterano de Palmas por meio do parecer número 85315418.0.0000.5516, os participantes foram convidados pessoalmente, de forma individual, de acordo com a disponibilidade de tempo de cada um. A cada abordagem realizada foi lido, junto ao participante, o Termo de Consentimento Livre e Esclarecido (TCLE), para clarear os objetivos da pesquisa e, mediante aceitação em participar, foram convidados a assinar o termo, conforme os princípios éticos da resolução do Conselho Nacional de Saúde (CNS) 466/12 (BRASIL, 2013).

A fim de preservar a identidade e sigilo, os participantes foram identificados por letras segundo sua categoria profissional, sendo a letra $E$ para os profissionais enfermeiros, $M$ para os médicos, $T$ para os técnicos de enfermagem e $A$ para os agentes comunitários de saúde, associadas por numeração arábica.

A coleta de dados ocorreu no período de abril a junho de 2018 pelo pesquisador responsável. Esta aconteceu nos CSCs selecionados em salas reservadas, diante da possibilidade de constrangimento e inibição ao responder às perguntas. Para isso foi aplicado um questionário semiestruturado desenvolvido pelos pesquisadores, realizado por meio de entrevista, com transcrição das falas dos participantes na íntegra. 0 questionário levantou informações gerais, como data de nascimento, profissão, tempo de atuação na saúde e o tipo de vínculo do profissional com a rede municipal de saúde. Do mesmo modo, abordou perguntas que tratavam da percepção do profissional acerca da $\mathrm{SM}$, como a respeito do conceito e diagnóstico, tratamento, fatores de risco e ações de prevenção.

Após a transcrição, foram identificadas as percepções e práticas relacionadas à SM, sendo as entrevistas analisadas pelo método de Análise de Conteúdo, conforme etapas descritas a seguir. O material foi analisado por meio de ampla leitura e distribuído em categorias (categorização) conforme agrupamento de ideias e expressões das falas dos profissionais por semelhanças. Após, foi realizada a inferência dos resultados obtidos e interpretação dos dados a partir da fundamentação teórica, buscando ressignificação aos conteúdos (BARDIN, 2011; MINAYO, 2014).

\section{RESULTADOS E DISCUSSÕES}

Foram entrevistados 4 técnicos de enfermagem, 4 agentes comunitários de saúde (ACS), 3 enfermeiros e 5 médicos, totalizando 16 profissionais. Destes, o tempo de atuação na saúde teve grande variação, sendo de 1 a 28 anos. Optou-se por questionar sobre o tempo de atuação e não quanto ao tempo de formado, pois os ACSs e técnicos de enfermagem não possuíam Graduação.

A média de idade dos entrevistados foi de 36,8 anos $\pm 9,59$. Com relação ao vínculo com o município, os técnicos de enfermagem, ACSs e enfermeiros, $100 \%$ eram profissionais efetivos. Quanto aos médicos, 20\% eram vinculados ao Programa Municipal Palmas para Todos, $20 \%$ efetivos e $60 \%$ residentes em Medicina de Família e Comunidade.

Conforme semelhança das falas, foram agrupadas para análise das entrevistas as categorias profissionais de enfermagem e medicina (Grupo 1) e ACS e técnico de enfermagem (Grupo 2), posto que o primeiro grupo estudou mais a fundo o tema. Para o mesmo fim, os resultados foram divididos em categorias, sendo elas: conceito e diagnóstico, tratamento, fatores de risco e ações de prevenção.

\section{Categoria 1: Conceito e Diagnóstico}

$\mathrm{Na}$ categoria definida como conceito e diagnóstico, observa-se que a resposta mais exteriorizada entre o grupo 2 foi que desconhecem a SM, além de a maioria demonstrar surpresa quanto ao uso do termo. Navarro et al. (2013) também cita o desconhecimento e dificuldade dos profissionais de saúde acerca do diagnóstico da SM. 
Não, eu acho que não entendo nada não, sinceramente. Eu nunca vi muito falar sobre isso (T2).

Uai, diagnóstico não é o médico que vai dá depois que ele ter a conclusão do coisa, da doença?! Também não entendo de diagnóstico (T3).

Não tenho entendimento (T4).

Síndrome metabólica? Não entendo nadinha (A2).

Tem a ver com saúde mental? Metabólica. Confesso, confesso que eu tenho que pesquisar no Google (A3).

Entre esse grupo, ainda nessa categoria, também houve uma repetida associação da SM com metabolismo, sendo possível observar que desconheciam o termo, porém tentavam fazer alguma ligação, sem aprofundamento, para não deixar de dar alguma resposta ao pesquisador, podendo demonstrar medo do erro e de assumir desconhecimento.

Síndrome metabólica? É do metabolismo da gente, né? É a dificuldade que tem o metabolismo? Eu entendo isso, que tem a ver com nosso metabolismo. A velocidade que ele funciona, isso (T1).

\section{[...] Tem alguma coisa a ver com metabolismo} (T2).

[...] Síndrome é uma doença e metabólica metabolismo né, mas eu não sei muito o que falar sobre isso não (T3).

Metabólico relacionado ao metabolismo da gente, é isso? (T4).

Apesar de surgir apenas uma vez, vale ressaltar que apenas um participante do Grupo 2 relacionou a SM à DM. "Ah, eu entendo assim, penso que é alguma coisa relacionada a diabetes" (A1). Da mesma forma, foi citado que para o diagnóstico é preciso a realização de exames. "Eu acho que tem que fazer exames" (A2).

Ainda na categoria de Conceito e Diagnóstico, o Grupo 1 demonstrou menos estranheza acerca do tema, tendo falas mais relacionadas à SM, porém ainda sem aprofundamento, conforme a literatura.

Síndrome metabólica? Olha, eu precisava estudar um pouco pra falar [...] É um distúrbio né, que ele tem que ser bem investigado né, pra poder a gente detectar e começar a trabalhar com o paciente, pra que ele não venha desencadear outras patologias em cima dessa síndrome né. Que no caso uma diabetes, uma hipertensão né, $e$ é isso (E1).
[...] Bom, eu acredito que ele, com certeza, deve ser feito através de exames né. Agora que tipo de exames. Glicemia, lipidograma. Imagino que seja mais ou menos isso. Através de exames de laboratório (E2).

Síndrome né? Então é um conjunto de sinais e sintomas né. Essa questão da síndrome metabólica, aí inclui que eu sei entra a questão, entra a hipertensão, diabetes, obesidade, a questão dos triglicérides, dos lipidogramas como geral. Mais ou menos em cima disso aí que eu mais ou menos eu sei, que é mais ou menos assim (E3).

Bom, o diagnóstico é um diagnóstico eminentemente clínico né, tem também a parte laboratorial para ajudar, mas assim, é basicamente um diagnóstico clínico, que a gente vai avaliar o paciente se ele tem hipertensão, diabete, faz o exame físico da cintura abdominal e aí o laboratorial a gente completa por triglicerídeo e HDL, então seria um clínico laboratorial, mas principalmente clínico, o diagnóstico (M1).

Toda doença que envolve diabetes, hipertensão, é, dislipidemia, tudo isso aí em relação aos, esses maus hábitos alimentares, que desenvolvem essas doenças crônicas né. Eu entendo por síndrome metabólica isso: hipertensão, diabetes, obesidade (M2).

Então, a síndrome metabólica é um conjunto né, de sinais, de sintomas, relacionados a alterações no sistema metabólico né. Pode ser diabetes, obesidade, hipercolesterolemia, enfim, é isso (M3).

Acho que é quando tem uma descompensação de vários fatores, por exemplo, a pessoa é diabético descompensado, tem alteração na parte de colesterol também, entendeu?! A gente tem que avaliar todo, não é só um critério (M4).

[...] É sobre fazer, tipo, glicemia de jejum, ver pelas questões do diabetes, acompanhamento de PA pra ver questão de hipertensão, de colesterol pra ver questão de dislipidemia, além das diversas né, não só essas (M5).

A SM pode ser conceituada como um conjunto de alterações metabólicas que exerce grandes fatores de risco cardiovascular associados ao aumento de circunferência abdominal, de glicemia, pressão arterial e alteração no metabolismo de lipídeos, podendo ter origem ambiental e genética, sendo relacionada ao desenvolvimento de DCNT com DCV e DM (SBC, 2005; IDF, 2006; OLIVEIRA; BOERY, 2018). 
Para diagnóstico da SM, de acordo com a I-DBSM (SBC, 2005), precisam estar presentes três dos cinco fatores apresentados, que são obesidade central (circunferência da cintura $>88 \mathrm{~cm}$ na mulher e $102 \mathrm{~cm}$ no homem), Hipertensão Arterial (pressão sistólica $\geq$ $130 \mathrm{mmHg}$ ou diastólica $\geq 85 \mathrm{mmHg}$ ), Glicemia de jejum ( $\geq 110 \mathrm{mg} / \mathrm{dl}$ ), Triglicerídeos ( $\geq 150 \mathrm{mg} / \mathrm{dl}$ ) e HDL colesterol $(<40 \mathrm{mg} / \mathrm{dl}$ em homens e $<50 \mathrm{mg} / \mathrm{dl} \mathrm{em}$ mulheres).

Ressalta-se ainda a importância do diagnóstico precoce para o manejo da SM na APS, pois, por estar mais próxima à comunidade, compreende melhor os determinantes sociais de saúde de seu território, podendo intervir nos fatores de risco e evitar que as DCNTs associadas se instalem, reduzindo a necessidade do uso de tratamento medicamentoso (BRASIL, 2014b).

\section{Categoria 2: Tratamento}

A categoria Tratamento obteve um padrão de resposta semelhante ao da primeira já referida anteriormente pelo Grupo 2, tanto referente ao desconhecimento da SM quanto à relacioná-la ao metabolismo. Uma resposta, apesar de única, porém, destacou-se por ter alguma relação com o tratamento, merecendo ser descrita: "Síndrome metabólica? Eu acho que o tratamento pode ser medicamentoso... E também pode ser, por questões de educação alimentar" (A2).

O grupo 1 demonstrou maior conhecimento e confiança para falar sobre o tratamento da SM. Apenas um profissional referiu desconhecer o tratamento. "Tratamento? Não faço ideia" (E2).

[...] Ai, tratamento você fala medicamentoso? Eu entro assim, a princípio, quando eu vejo que tem alguma alteração eu vou trabalhar aquela parte de orientação alimentar, atividade física. Agora quando já tem que entrar com medicamento né, que é já a parte de médica (E1).

Vai ser em cima do que tiver das alterações né, na síndrome. Então se for uma questão lipidogra$\mathrm{ma}$, se for uma hipertensão, se for uma diabetes. A obesidade, ela já é mais ampla né, que aí já vai mexer com uma série de outros problemas também (E3).

Como grande parte do problema da síndrome metabólica tá relacionado com alimentação, não adianta a gente querer mudar só o perfil do paciente com síndrome metabólica se ele não mudar a rotina familiar [...] Então seria o tratamento, é, tanto pessoal, tratamento familiar e que algumas vezes pode ser necessário também o, os medicamentos, mas principalmente tem que ser corrigida a dieta da pessoa inicialmente (M1).

Vai desde medicamentoso até mudança de hábito né, mudança, mudanças de hábito mesmo. Mudança de hábito alimentar, estilo de vida (M2).

O tratamento da síndrome ele requer um amplo, uma ampla gama de pessoal pra gente conseguir fazer né, vários profissionais: nutricionista, médi$c o$, enfim, pra que a gente consiga tratar todas as causas que estão levando à sindrome (M3).

Que é um tratamento que depende de, não só de medicação, mas da alteração do estilo de vida do paciente (M4).

[...] fazer um tratamento certo, um tratamento correto que o paciente ajude no, nesse tratamento, que ele tenha todo conhecimento da medicação, isso aumenta bastante a sobrevida dele (M5).

O tratamento consiste, principalmente, em meIhora do estilo de vida, considerando a mudança de hábito alimentar, tendo em vista a qualidade da alimentação e a quantidade de alimentos ingeridos, visando à perda de peso, sendo esta associada a exercício físico regular (SBS, 2005). Para tanto, intervenções multidisciplinares, tanto individuais quanto em grupos, podem ser utilizadas e demonstram redução nos parâmetros da SM em estudos (SOARES et al., 2014; SABOYA et al., 2017).

A APS é um local privilegiado para a realização das ações referidas, por possuir apoio de equipe multiprofissional, ser coordenadora do cuidado e centro de comunicação entre os demais pontos de atenção da rede de atenção à saúde, a fim de garantir o cuidado integral do usuário (BRASIL, 2017b). O tratamento medicamentoso pode ser utilizado em alguns casos específicos (SBC, 2005; ABESO, 2010).

\section{Categoria 3: Fatores de Risco}

Ao analisar a categoria sobre os fatores de risco, foi possível destacar que a maioria dos entrevistados do grupo 2 referiram não ter conhecimento acerca do assunto, como relatado: "Como que eu vou responder se eu não sei nadinha desse negócio" (T3) e "Sinceramente não sei que é fundamentar. Teria que estudar realmente" (A3).

Dois dos profissionais, no entanto, conseguiram opinar e relacionar a SM a alguns fatores de risco relevantes: "Pode ser uma questão também genética, né. 
Ou pode ser também questão da má alimentação [...]" (A2). "Pode ser obesidade, as doenças que vem com a obesidade [...]" (T1).

O Grupo 1, de forma geral, fez levantamentos importantes acerca dos fatores de risco para desenvolvimento da SM, aproximando-se ao encontrado na literatura, demonstrando maior entendimento sobre a questão proposta.

Ah, é, os fatores de risco acho que também entra um pouco da genética (E1).

Tem a questão genética, tem os hábitos de vida, né. Que nos hábitos de vida entra a questão da alimentação né, do sedentarismo, o estilo de vida mesmo, da pessoa (E3).

[...] engloba os hábitos alimentares da pessoa, atividade física ou não. Nesse quesito a gente tem que ver bem a alimentação, tanto quantitativamente quanto qualitativamente, o que ele tá ingerindo. Tabagismo, o etilismo, que faz, é, faz diferença também [...] (M1).

[...] Maus hábitos alimentares, que é outro fator de risco, a hipertensão, a diabetes é, sedentarismo, tabagismo, etilismo, tudo isso vai entrar né, na síndrome metabólica (M2).

Tem os fatores que influenciam a parte genética do paciente e do estilo de vida mesmo (M4).

Tabagismo, etilismo, sedentarismo. Todos esses levam a síndromes metabólicas né, em geral. Má alimentação, isso aí (M5).

A SM pode ter influência de fatores ambientais e genéticos (OLIVEIRA; BOERY, 2018). De acordo com a literatura, os principais fatores de risco para seu desenvolvimento são o excesso de peso, os hábitos alimentares inadequados e o sedentarismo, podendo ressaltar ainda o tabagismo e nível socioeconômico (JARAMILLO et al., 2014; PONTES; AMORIM; LIRA, 2016).

\section{Categoria 4: Ações de Prevenção}

Ações de prevenção e promoção da saúde são preconizadas desde políticas públicas, como a Política Nacional de Atenção Básica, até os Cadernos de Atenção Básica voltados às DCNTs e obesidade e o MACC (MENDES, 2012; BRASIL, 2014b, 2014c, 2017b). Na categoria estabelecida sobre ações de prevenção da $\mathrm{SM}$, porém, quando questionados se havia alguma desenvolvendo-se em seu CSC, houve muitas respostas semelhantes e em quantidades parecidas, tanto do
Grupo 1 quanto no Grupo 2, destacando não haver ou desconhecerem ações de prevenção em desenvolvimento.

Falas aleatórias, entretanto, de algumas atividades conduzidas atualmente ou que já foram realizadas, foram levantadas, demonstrando um certo conhecimento acerca da promoção e da prevenção.

Tem, com nutricionista né?! Que se tem o atendimento da nutrição, que orienta. Tem esse. Antes a gente tinha mais os grupos, de caminhada, essas coisas era mais ativo. Tinha um liang gong, também, que a população tinha como participar junto com a Unidade. Hoje não tem mais (T1).

Seria as atividade na sala de espera que vocês fazem aqui? (T3).

Sim, acredito que tem (A1).

A prevenção não. Trabalho em grupo não. Mas tem a parte da nutricional, $a$, o nosso atendimento do dia a dia né, que a gente orienta muito (E1).

Sim, exatamente uma coisa que eu não sabia até pouco tempo de estudar um pouquinho mais sobre o próprio PSE [Programa Saúde na Escola], a nossa intervenção lá na infância, quando a gente como saúde vai nas escolas trabalhar esses fatores já [...] Na verdade desde a gestação, então começa assim, no pré-natal nosso, na orientação da mãe, na puericultura, mais especificamente [...] A questão da prevenção nós temos o saúde do homem, né. A própria saúde da mulher. Saúde do homem esses eventos pontuais assim, semestrais que a gente faz ocasionalmente e onde a gente faz essa avaliação da própria triagem; a gente já, já aparece alguns casos suspeitos. A gente vai dar uma atenção maior [...] (E3).

Bom, atualmente, é, pelo menos na minha equipe, não tá tendo nenhuma, é, nenhuma ação. $A$ gente tinha o grupo de hipertenso e diabético, que a gente fazia o esclarecimento, sobre síndrome metabólica. No grupo do homem a gente já teve esse assunto durante três vez, onde a gente fez medida da cintura abdominal, passou as orientações, então, assim, a que a gente tinha era mais vindo de conscientização. Atualmente, já tem uns cinco, seis meses que nós estamos sem nada, é, voltado especificamente pra isso, fica mais aquela ação de consultório. $O$ paciente identificado a gente tem $o$, a gente faz $o, a$, todos os esclarecimentos necessários, entendeu, mas assim, aquilo de prevenção hoje tá realmente em falta (M1). 
Eles falam pra gente fazer uma palestra aqui, agora eu acho que vai começar a ter, porque dentro dessas palestras os que a gente tem que pegar mais são esses pacientes né. Aí eu acho que vai começar a ter agora semanalmente (M5).

Nota-se, a partir das respostas, que os profissionais possuem conhecimento acerca da prevenção da SM e sua importância, porém essas ações estão sendo cada vez mais infrequentes nos CSCs, contrapondo com o que é preconizado pelas políticas de saúde. Sabe-se que quando o usuário chega ao CSC já apresenta algum quadro de agravamento de doenças, demonstrando possível falha no diagnóstico precoce e ações de prevenção na APS voltadas aos fatores de risco associados (BRASIL, 2014b). Uma maior investigação do motivo da redução de tais ações deve ser realizada para levantamento de problemas e propostas de soluções, posto que atividades voltadas à promoção e à prevenção podem contribuir para a diminuição do surgimento de patologias como a SM.

Quando questionados se gostariam de complementar com mais alguma informação houve, no grupo 2 , respostas que sugerem o interesse por buscar saber mais sobre o assunto, ainda considerando que os envolvidos já passaram por capacitações iniciais acerca do tema.

Quero saber o que é isso aí. Agora fiquei curiosa (T3).

Eu gostaria de saber o que que era. $O$ que que isso é relacionado (T4).

[...] acredito que se nós, poderíamos até ter sobre essa aí [...] Ter conhecimento sobre o estudo e uma educação continuada sobre a síndrome metabólica e ainda não temos, infelizmente. Eu não tenho conhecimento. Não tive ainda. (A3).

Eu queria era saber, do que nós estamos falando (A4).

Esse achado corrobora o estabelecimento do MACC, pois, além de sugerir que o tema seja abordado com esses profissionais utilizando uma metodologia diferente, também ressalta que, quando se é despertada a curiosidade e a busca por conhecimento, o processo de aceitação do novo modelo de atenção é facilitado em virtude da importância que os envolvidos darão ao tema.

Sugere-se, ainda, como solução do problema encontrado, capacitações de educação permanente com todos os profissionais da APS voltadas à SM. Como limitante da pesquisa pode-se destacar a falta de estudos que avaliem a percepção dos profissionais sobre o tema. Outro fator limitador foi a não publicação de documentos, até o momento, voltados ao MACC no município com o foco na SM.

\section{CONCLUSÃO}

Os profissionais de nível superior demonstraram maior compreensão acerca da SM, porém ainda nota-se a necessidade de mais esclarecimentos sobre o assunto para que a importância desta condição seja reconhecida. Desta forma, haverá uma prestação de assistência adequada aos pacientes, evitando o surgimento de novos casos de síndrome metabólica e que doenças crônicas não transmissíveis se instalem, causando consequências econômicas e sociais para o país.

Os resultados desta pesquisa permitem aos gestores tomadas de decisão orientadas por evidências para desenvolver ações de educação permanente aos profissionais de saúde acerca do tema, obtendo como consequência a melhoria na qualidade dos cuidados ofertados à população.

Além disso, o estudo instigou a curiosidade sobre o tema pelos entrevistados, facilitando a aceitação e o processo de instalação do novo modelo de atenção - o Modelo de Atenção as Condições Crônicas -, que está sendo proposto no município. Assim, favorece tanto os profissionais participantes da pesquisa quanto a gestão e toda a comunidade indiretamente.

\section{REFERÊNCIAS}

ABESO. Associação Brasileira para o Estudo da Obesidade e da Síndrome Metabólica. Atualização das diretrizes para o tratamento farmacológico da obesidade e do sobrepeso. Posicionamento oficial da Abeso/SBEM - 2010. Edição especial. Abeso, 2010. $15 \mathrm{p}$.

ABESO. Diretrizes brasileiras de obesidade 2016. 4. ed. São Paulo: Abeso, 2016. 188 p.

BARDIN, L. Análise de conteúdo. 6. ed. São Paulo: Edições 70, 2011. 279 p.

BRASIL. Conselho Nacional de Saúde. Resolução $n^{\circ} 466$, de 12 de dezembro de 2012. Aprova normas regulamentadoras de pesquisas envolvendo seres humanos. Brasília: Diário Oficial da União, 2013.

BRASIL. Pesquisa Nacional de Saúde 2013: percepção do estado de saúde, estilos de vida e doenças crônicas. Brasil, grandes regiões e unidades da federação. Rio de Janeiro: Ministério do Planejamento, Orçamento e Gestão; Instituto Brasileiro de Geografia e Estatística; Diretoria de Pesquisas; Coordenação de População e Indicadores Sociais, 2014a. $181 \mathrm{p}$. 
BRASIL. Estratégias para o cuidado da pessoa com doença crônica. Brasília: Ministério da Saúde; Secretaria de Atenção à Saúde; Departamento de Atenção Básica, 2014b. 162 p.

BRASIL. Estratégias para o cuidado da pessoa com doença crônica: obesidade. Brasília: Ministério da Saúde; Secretaria de Atenção à Saúde; Departamento de Atenção Básica, 2014c. 212 p.

BRASIL. Relatório do estado nutricional dos indivíduos acompanhados por período, fase do ciclo da vida e índice. Brasília: Ministério da Saúde; Secretaria de Assistência à Saúde; Departamento de Atenção Básica; Núcleo de Tecnologia da Informação; Sistema de Vigilância Alimentar e Nutricional - Sisvan. 2016a. Disponível em: http://dabsistemas.saude.gov.br/sistemas/sisvan/relatorios_publicos/ relatorio-acomp-nutri.view.php. Acesso em: 23 nov. 2017.

BRASIL. Pesquisa Nacional de Saúde do Escolar 2015. Rio de Janeiro: Ministério do Planejamento, Orçamento e Gestão; Instituto Brasileiro de Geografia e Estatística; Diretoria de Pesquisas; Coordenação de População e Indicadores Sociais, 2016b. 131 p.

BRASIL. Vigitel Brasil 2016: vigilância de fatores de risco e proteção para doenças crônicas por inquérito telefônico: estimativas sobre frequência e distribuição sociodemográfica de fatores de risco e proteção para doenças crônicas nas capitais dos 26 Estados brasileiros e no Distrito Federal em 2016. Brasília: Ministério da Saúde. Secretaria de Vigilância em Saúde. Departamento de Vigilância de Doenças e Agravos não Transmissíveis e Promoção da Saúde, 2017a. 160 p.

BRASIL. Portaria no 2.436, de 21 de setembro de 2017. Aprova a Política Nacional de Atenção Básica, estabelecendo a revisão de diretrizes para a organização da Atenção Básica no âmbito do Sistema Único de Saúde (SUS). Brasília: Ministério da Saúde, 2017b. Disponível em: http://bvsms.saude.gov.br/bvs/saudelegis/gm/2017/prt2436_22_09_2017. html. Acesso em: 10 nov. 2017.

CASTANHO, G. K. F. et al. Consumo de frutas, verduras e legumes associado à síndrome metabólica e seus componentes em amostra populacional adulta. Ciência \& Saúde Coletiva, v. 18, n. 2, p. 385-392, 2013.

IDF. International Diabetes Federation. The IDF consensus worldwide definition of the metabolic syndrome. Brussels: IDF, 2006. 24 p.

JARAMILLO, P. L. et al. Consenso latino-americano de hipertensão em pacientes com diabetes tipo 2 e síndrome metabólica. Arquivo Brasileiro de Endocrinologia e Metabologia, v. 58, n. 3, p. 205-225, 2014.

MENDES, E. V. O cuidado das condições crônicas na atenção primária à saúde: o imperativo da consolidação da estratégia da saúde da família. Brasília: Organização Pan-Americana da Saúde, 2012. 512 p.

MINAYO, M. C. S. O desafio do conhecimento: pesquisa qualitativa em saúde. 12. ed. São Paulo: Hucitec Editora, 2014.

MOYSÉS, S. T.; FILHO, A. D. S.; MOYSÉS, S. J. Laboratório de inovações no cuidado das condições crônicas na APS: a implantação do modelo de atenção às condições crônicas na UBS Alvorada em Curitiba. Brasília: Organização Pan-Americana da Saúde, 2012. 198 p.
NAVARRO, N. et al. Vocabulário padronizado e informações em saúde: é necessária uma codificação para a síndrome metabólica. Revista Eletrônica de Comunicação, Informação \& Inovação em Saúde, Rio de Janeiro, v. 7, n. 2, 11 p., 2013. OLIVEIRA, J. C.; BOERY, R. N. S. O. Associação de variantes polimórficas com síndrome metabólica: uma revisão integrativa. Jornal Vascular Brasileiro, v. 17, n. 2, p. 141-147, 2018.

PALMAS. Secretaria Municipal de Saúde. Portaria Inst no 518 de 14 de junho e 2016. Institui a Rede de Atenção e Vigilância em Saúde de Palmas. Diário Oficial do Município de Palmas, Palmas, n. 1.533, p. 12-14, 2016. Disponível em: http://diariooficial.palmas.to.gov.br/media/diario/1533-28-6-2016-18-47-29.pdf. Acesso em: 21 jan. 2018. PONTES, L. M.; AMORIM, R. J. M.; LIRA, P. I. C. Componentes da síndrome metabólica e fatores associados em adolescentes: estudo caso-controle. Revista da AMRIGS, Porto Alegre, v. 60, n. 2, p. 121-128, 2016.

SAAD, M. S. N. et al. Prevalência de síndrome metabólica em idosos e concordância entre quatro critérios diagnósticos. Arquivo Brasileiro de Cardiologia, v. 102, n. 3, p. 263269, 2014.

SABOYA, P. P. et al. Intervenção de estilo de vida na síndrome metabólica e seu impacto na qualidade de vida: um estudo controlado randomizado. Arquivo Brasileiro de Cardiologia, v. 108, n. 1, p. 60-69, 2017.

SBC. Sociedade Brasileira de Cardiologia. I Diretriz brasileira de diagnóstico e tratamento da Síndrome Metabólica. Arquivo Brasileiro de Cardiologia. São Paulo, v.84, suppl. 1, 28 p., abr. 2005.

SBD. Sociedade Brasileira de Diabetes. Diretrizes Sociedade Brasileira de Diabetes 2017-2018. São Paulo: Editora Clannad, 2017.

SOARES, T. S. et al. Hábitos alimentares, atividade física e escore de risco global de Framinghan na síndrome metabólica. Arquivo Brasileiro de Cardiologia, v. 102, n. 4, p. 374382, 2014.

WHO. World Health Organization. Definition, diagnosis and classification of diabetes mellitus and its complications. Part 1: Diagnosis and classification of diabetes mellitus, department of non communicable disease surveillance. Geneva: WHO, 1999. $66 \mathrm{p}$. 